\title{
A Method for Real-Time Aggregation of a Product Footprint during Manufacturing
}

\author{
Peter Smolek $^{* 1}$, Ines Leobner ${ }^{2}$, Bernhard Heinzl, Georgios Gourlis ${ }^{4}$, Karl Ponweiser ${ }^{5}$ \\ ${ }^{1}$ Institute for Energy Systems and Thermodynamics, TU Wien, Getreidemarkt 9, 1060 Vienna, Austria \\ e-mail: peter.smolek@tuwien.ac.at \\ ${ }^{2}$ Institute for Energy Systems and Thermodynamics, TU Wien, Getreidemarkt 9, 1060 Vienna, Austria \\ e-mail: ines.leobner@tuwien.ac.at \\ ${ }^{3}$ Institute of Computer Aided Automation, TU Wien, Treitlstr. 1-3, 1040 Vienna, Austria \\ e-mail: bernhard.heinzl@tuwien.ac.at \\ ${ }^{4}$ Institute of Interdisciplinary Construction Process Management, TU Wien, Karlsplatz 13, \\ 1040 Vienna, Austria \\ e-mail: georgios.gourlis@tuwien.ac.at \\ ${ }^{5}$ Institute for Energy Systems and Thermodynamics, TU Wien, Getreidemarkt 9, 1060 Vienna, Austria \\ e-mail: karl.ponweiser@tuwien.ac.at
}

Cite as: Smolek, P., Leobner, I., Heinzl, B., Gourlis, G., Ponweiser, K., A Method for Real-Time Aggregation of a Product Footprint during Manufacturing, J. sustain. dev. energy water environ. syst., 4(4), pp 360-378, 2016, DOI: http://dx.doi.org/10.13044/j.sdewes.2016.04.0028

\begin{abstract}
To assess cost, time investment, energy consumption and carbon emission of manufacturing on a per-piece basis, a bottom-up approach for aggregating a real-time product footprint is proposed. This method allows the evaluation of the environmental impact of a batch or even single product using monitoring or simulation data. To analyze the infrastructure, the production plant is decomposed into modules that are in relation to each other via inputs and outputs. Distinguishing between modules for production, logistics, energy system, buildings and auxiliary systems, the different approaches for distributing resource consumption between the products are presented. Special attention is paid to typical scenarios that occur in production plants and problems that may arise from them. For example, the incorporation of standby-, setup- and ramp-up times, the energy consumption of the administration and the allocation of different products and by-products manufactured at a machine are taken into account.
\end{abstract}

\section{KEYWORDS}

Energy efficient industry, Simulation, Carbon footprint of products, Product life-cycle assessment, Manufacturing.

\section{INTRODUCTION}

Industrial production accounts for $26 \%$ of the energy consumption of Europe [1], with an estimated potential for reduction of $30 \%$ to $65 \%$ [2]. A commonly used benchmark to identify the environmental impact of products is to assess the Carbon Footprint of Products (CFP) on a one-year-basis. A variety of case studies concerning life-cycle assessment including carbon footprints has been published in literature for all different kinds of products and services, such as refrigerators [3], domestic water heating devices [4], urban transport systems [5] or even scientific publications [6]. The footprint-procedure is important for raising awareness and providing decision making guidelines on the customer side as well as within the company. [7] showed that awareness of the management is one of the factors significantly affecting the CFP in companies.

\footnotetext{
* Corresponding author
} 
However, surveys of currently available standardizations and tools have brought up some issues concerning the reliability of results due to the lack of transparency of different methods [8], standardization [9] and reliable data [10].

The usual top-down approaches for calculating footprints are even less suited to support sustainable plant operation, because they lack the temporal and spatial resolution necessary to base resilient statements upon them. [11] concludes, that one aggregated indicator is essential for communication but failing to provide the required detail to undertake a meaningful assessment of the energy use within the production system. In recent years several publications have introduced methodologies to integrate energy efficiency into production planning, such as $[12,13]$. In order to meet the demand of these approaches, footprints need to include both, aggregated and decomposed figures. While the static aggregate may remain roughly the same over time, the contributions to it may change significantly. Integrating these features could transition carbon footprints from being a static analysis tool to a continuous process assessment and steering measure. [14] meets this demand by providing a method for assessing a time-resolved footprint of the supplied energy. [15] attempts to integrate a higher resolution into product footprints by means of simulation. However, [16] stresses the industry's need for real-time data and knowledge-embedded processes leading to significant Key Performance Indicators (KPI) of energy efficiency. A CFP of adequate temporal and spatial resolution could serve this purpose as well as be a means of communication to stakeholders.

In order to address these issues, a bottom-up approach for aggregating a product footprint during the production phase (see Figure 1) of the product life-cycle is proposed. This method allows for real-time evaluation of a batch or even single product using monitoring and/or simulation data. The definition of a significant footprint sets product success factors in context with its ecological impact. In particular, energy, costs, carbon emission and time will be captured and visualized for the whole transformation process a product undergoes within the plant. Each part of the plant contributes to the product's energy, cost or time consumption, as well as carbon emission, which accumulate the product footprint. The energy used by production machines, auxiliary infrastructure, logistics and the building is aggregated from the entry of raw materials to the departure of finished goods. The integral footprint of all products produced in a year exactly matches the yearly carbon footprint of the plant. Therefore comparability with conventional studies is achieved.

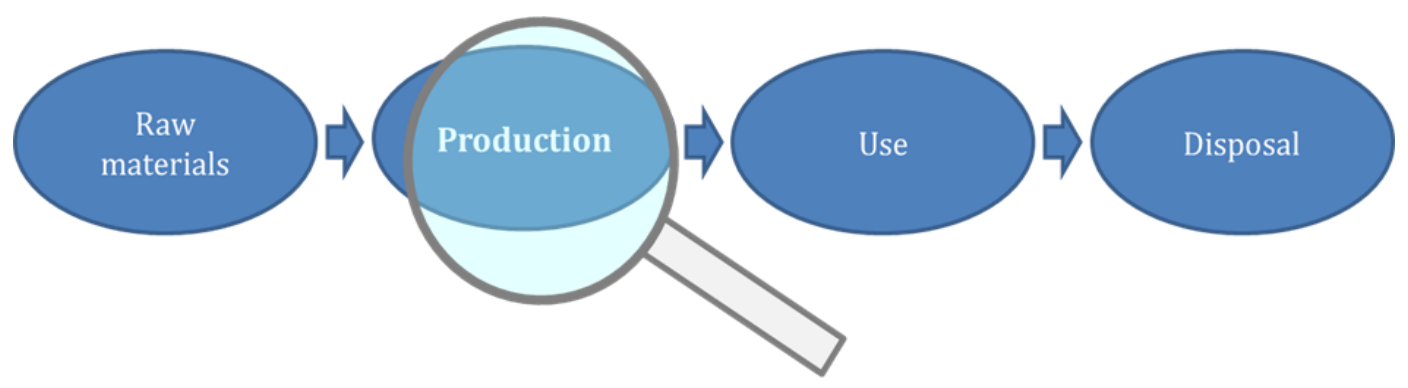

Figure 1. Different stages in the life-cycle of a product

From a bottom-up approach different challenges arise. For example, incorporation of standby-, setup- and ramp-up times, energy consumption of the administration and allocation of different products and by-products manufactured at the same machine are some of the problems. The necessity to calculate mean values and distribute them among different products demands for a method to assess the degree of which each product is responsible for the generated footprint. 
In the next section the methods are presented in detail. First the necessary definitions are made and the boundaries for application of the methods are discussed. The decomposition of the system, as well as the modelling approach using "cubes", is described and the specific calculations for assessing the product footprint are shown. To further clarify the methods a showcase is presented. Finally, the conclusions are drawn.

\section{METHODS}

A product footprint is defined in order to assess the impact of product manufacturing on the environment and to set the product success factors in context with its ecological impact. The considered expenditures are energy, cost, carbon emission and time. In general for defining a footprint, investments are aggregated over the entire life-cycle of a product, from the "cradle" to the "grave". The life-cycle can be divided into four phases during a product's lifetime, starting from the acquisition of resource materials, continuing with the manufacturing process and product usage and finally ending with its disposal and recycling. The classification of the different kinds of footprints is shown in Figure 2. The product life-cycle footprint is the total footprint during the lifetime of the product. It can be classified by the four components of the footprint and by the phase in the product life-cycle. According to ISO 14067, the carbon emission footprint during the life-cycle of the product is equivalent to the CFP as defined in the standard.

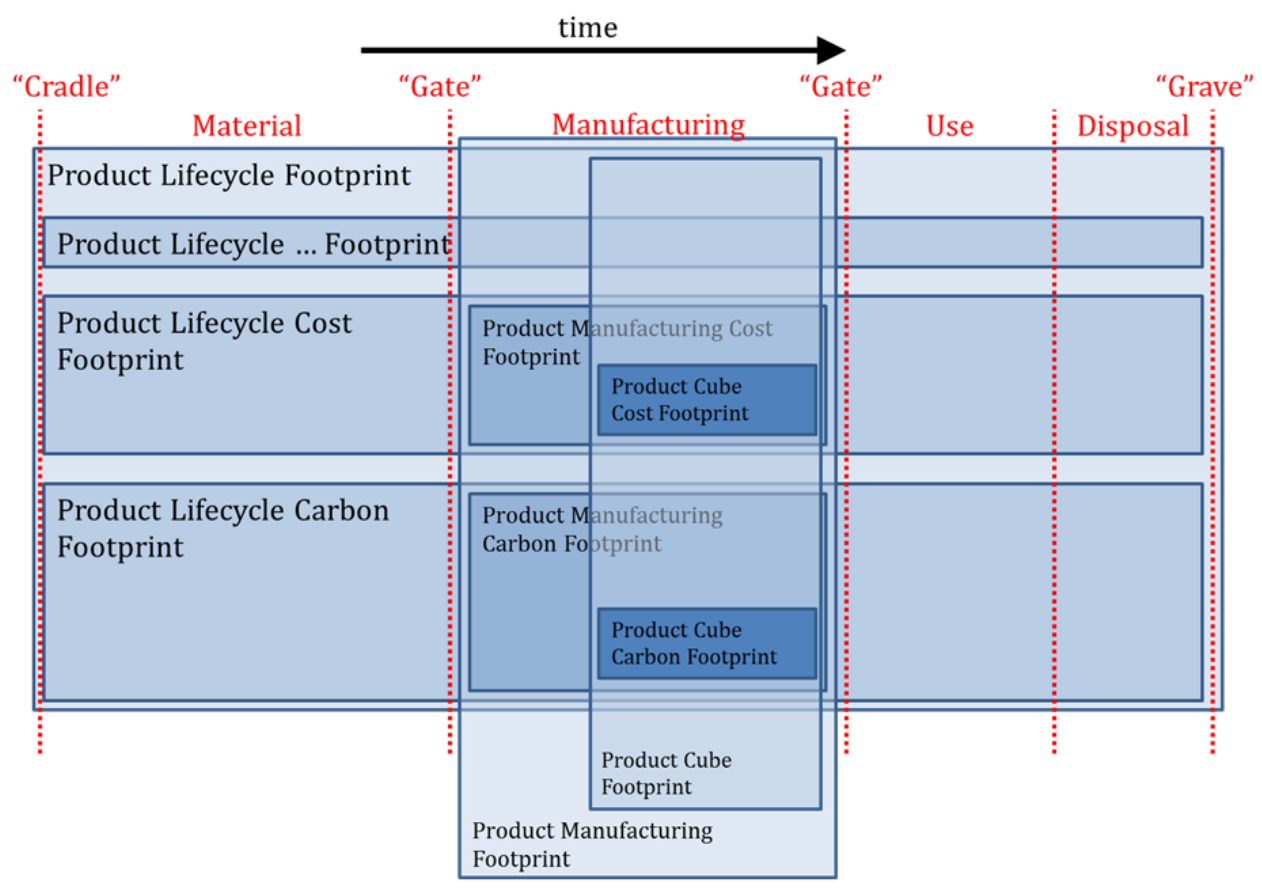

Figure 2. Classification of product footprints

In this research, the product footprint during the production phase of a product's life-cycle is the main focus. This footprint can be called product manufacturing footprint and is a partial footprint using the gate-to-gate approach. This allows for a detailed assessment of the manufacturing processes and a reliable calculation of the footprint aggregated during production. In order to achieve the necessary level of detail, a bottom-up approach is used. Each process in a production plant is determined and modelled in a hierarchical way. On the process level, balances regarding footprint calculations are implemented. The result is a framework which integrates the connections between individual processes and captures the complexity of the entire production plant. 
This allows attributing the product footprint, from gate to gate, separately to each manufactured product.

\section{Application}

The product footprint application area is focused on the manufacturing phase of a product's life-cycle, however it could be extended outside this limit and increase its level of detail when appropriate information is available. When widening the scope of the application, principles and conditions defined in this paper shall be respectively applied to all parameters and systems considered. Furthermore the proposed product footprint aggregation method can be utilized in existing industrial facilities as well as in virtual environments, such as that of a planning and simulation system of a future plant.

\section{Gate-to-gate approach}

In assessing the environmental impact of a product throughout its entire life-cycle (product life-cycle footprint), analyzing the production phase is only a part of it, although a very important one because of its high energy input and related $\mathrm{CO}_{2}$ emissions in general.

An inclusion of the entire product life-cycle would require qualified assumptions about raw materials, transport, usage, disposal and other life-cycle phases, therefore an arduous task when considering that these stages are affected by day-to-day fluctuations, being beyond the scope of this paper.

For this reason, the focus lies on a gate-to-gate analysis of the production phase inside a considered production facility and the development of a method for a more detailed calculation of the product manufacturing footprint. This footprint can later be extended by incorporating other life-cycle phases in order to obtain a life-cycle footprint. On the other hand, if only the overall life-cycle footprint was reported, it would not be possible to extract individual manufacturing shares since information is lost during aggregation, explaining why it is not always the best idea to only focus on overall product life-cycle footprint considerations.

A gate-to-gate product manufacturing footprint is useful among other things for product-related comparison between different production scenarios or optimization strategies. For example, when calculating the difference between life-cycle footprints of two production scenarios, footprint shares outside the production process are not relevant because they cancel each other out in the subtraction (assuming the same conditions). This allows making relative comparisons of production strategies based on the product-related environmental impact or to use the manufacturing footprint as possible target function for optimization of operational management strategies.

However, one must be careful when trying to compare absolute values of the product manufacturing footprint to other (non-manufacturing) footprint values, especially if they are based on other calculation methods, because they may include different stages of the life-cycle. It is still possible to use the manufacturing footprint for such a comparison, but it has to be extended by making the same assumptions about transport, use, disposal, etc. and adding respective shares to the footprint in order to ensure a fair result.

\section{Static and dynamic footprint}

There are two parts that affect the overall product footprint, the dynamic and the static footprint. The dynamic footprint consists of the cost, energy demand and the emission of $\mathrm{CO}_{2}$ equivalent during the product's time inside the manufacturing plant and is calculated from the consumption of energy (for production process and indoor climate conditions), media, materials and discards, as well as the energy required for transportation. In 
contrast, the static carbon footprint is the cost, embedded energy demand and emission of $\mathrm{CO}_{2}$ equivalent needed for supplying the means of production. It is calculated from the materials and components in the building constructions, Heating, Ventilation, Air Conditioning (HVAC) systems and the manufacturing machines. After the initial investment the static footprint is a fixed value until future additions to the facility are made. However, values for cost, energy and $\mathrm{CO}_{2}$ depreciate over the planned service time of installed machinery or the building systems and constructions, therefore parallel methods for controlling and adding the static footprint portion to respective products have to be implemented.

If reliable data is available, the static product footprint can be taken into consideration. This requires a carbon and energy footprint value of the production machines for the operational period during which the general product footprint calculation is performed. The machinery static footprint would then be calculated on an hourly rate. The static footprint of building elements could also be considered on such a basis.

Taking the above into consideration, at this stage the documented method will address footprint calculations only in terms of dynamic carbon footprint.

\section{System boundary}

The system boundary determines the investigation area and defines which systems, processes, facilities, etc. are to be included within the product footprint study. It sets clear limits between the analyzed system and the surrounding environment as well as the conditions according to which interactions occur. As already mentioned, the goal is to aggregate a consistent footprint of the production phase during the product's life-cycle and therefore the system boundary is defined following a gate-to-gate approach. All material, energy and resource flows that enter or exit the system must be considered.

Decisions shall be made regarding which units of the provided infrastructure are to be included in the analysis and to which level of detail these units shall be studied. The selection of cut-off criteria used for the system boundary establishment, shall be identified, clearly stated and explained. In case the system boundary refers to a partial procedure and omits processes, facilities and sub-systems that in general interact with each other, the reasons and criteria of such a practice must be explained.

\section{Time boundary}

In principle, the time boundaries can be set arbitrarily. One advantage of a real-time footprint aggregation is the independence of a fixed time frame. The footprint for all products, by-products and waste can be calculated for the chosen period. This requires the knowledge of the initial footprint values at the beginning of the observation period. For recurring analysis, these values are known. If this is not the case, the starting point of the analysis has to be set to an earlier time to incorporate the entire stay of the product of interest inside the plant.

For recurring analysis of the product footprint, a fixed time frame helps with consistent results, as this issue arises out of the need to calculate mean values. To sustain a satisfying level of comparability, some compromise has to be made. Considering a single day as a timeframe, it becomes obvious that differences between weekends and weekdays exist. Comparing the footprint of a product manufactured on a weekday would most likely yield higher values due to administration and other energy consumers only present on a workday. The impact of these differences depends on the individual case.

As a compromise between comparability and short time periods, the time span of a week is proposed as a standard time boundary, starting on Monday 00:00 and ending on 
Sunday 24:00 local time. Longer time periods in the form of multitudes of one week can be used trouble-free. Shorter periods may lead to fluctuating results. For future references the standard time period of one week is applied. If a different time period is used in application a thorough justification is required.

It has to be mentioned, that the time period of one week is not exempt from comparability problems explained above. For example holidays can lower the amount of productive days of a week and distort the results. Therefore, the impact of these effects on the individual case has to be studied.

\section{Decomposition of the system}

The Life-Cycle Inventory analysis (LCI) as defined by the ISO 14067 standard is the process of compiling and quantifying the inputs and outputs for a product throughout its life-cycle. Part of the LCI is the data collection, quantification and allocation of flows. In the scope of the presented method, the LCI can be interpreted as the deconstruction of the facilities inside the system boundary into modules that are in relation to each other via inputs and outputs. These modules are referred to as "cubes". The entire system is then constructed using cubes, identifying the material, energy and information flows between them. Figure 3 shows an example of a plant represented by cubes. Inside the system boundary, the plant is divided into two thermal zones (green). Inside the thermal zones are production equipment (blue), transport and storage facilities (purple), energy systems (red) and administration (grey). The arrows represent the input/output relations between the cubes.

Through all of the cubes in the example, the product that follows the value flow is either directly or indirectly affected.

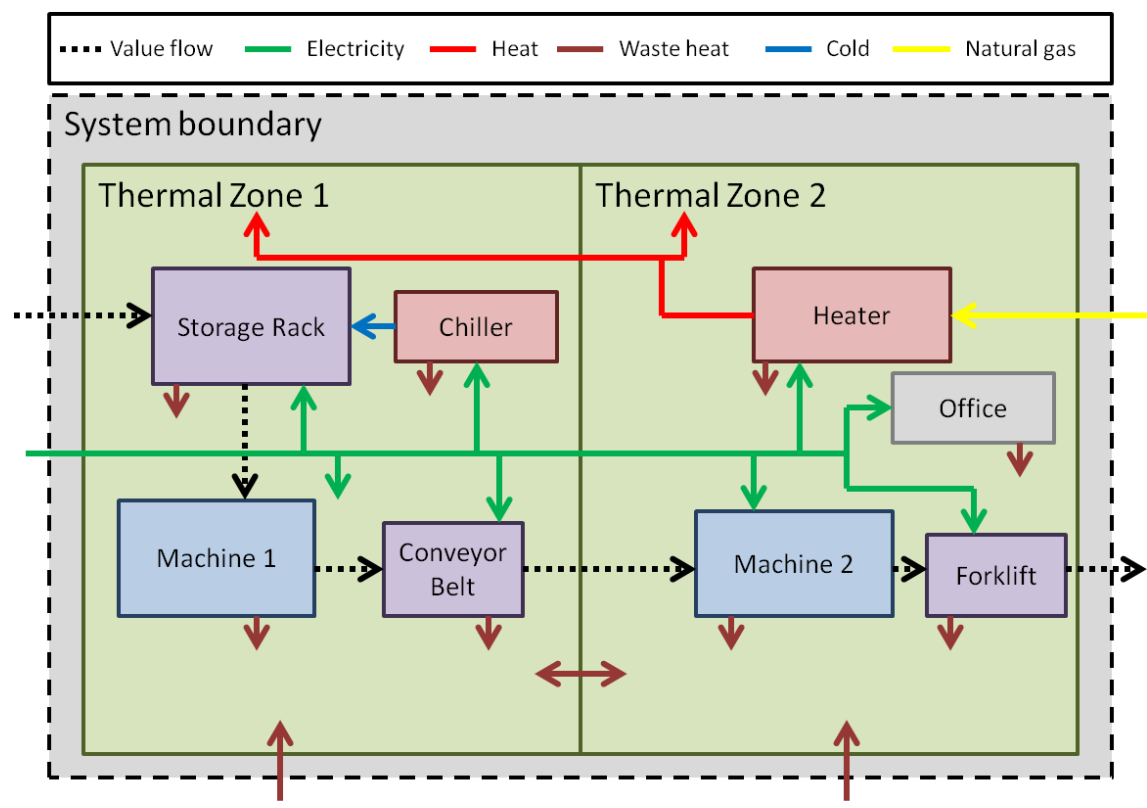

Figure 3. Example of cubes' relations within a plant

\section{Cubes}

The generic term "Cube" describes an encapsulated part of the observed overall system. This is part of a methodical approach (as found in [17]) to address the high system complexity and heterogeneity by dividing the overall system from an energetic point of view into well-defined manageable modules (see Figure 3), which then allow a focused system analysis independent of the surrounding environment. Cube instances are 
connected via their interfaces, therefore mapping the topology of the system under consideration. Cubes themselves can in turn be composed of other cubes (composite cube), thereby enabling hierarchical structuring of the overall system. For example, a composite cube may consist of a machine cube with local input queue (logistics cube), and itself be located within a thermal zone cube. The internal details of the composite cube are in many cases not relevant to the outside, so it is sufficient to represent the composite cube as a black box on a higher level, thereby hiding unnecessary model complexity and gaining clarity. In the same way, composite cubes also allow iterative model development with subsequent refinement of specific sections depending on the necessary level of detail without influencing the surrounding cube models.

Concerning the product footprint, energy, time, cost and $\mathrm{CO}_{2}$ shares are either accumulated and directly assigned to the product value stream currently inside the cube, or, if this is not possible, handed to the cube in the hierarchy above where calculations for footprint values, which cannot directly be attributed to individual products, are made. Integrating different viewpoints and areas of engineering (machinery, energy system, building, and logistics) in a single system description makes it necessary to establish a general specification of the cube properties and interfaces.

A detailed documentation about cubes, explaining all their aspects and characteristics, can be found in [17] and the documentation of Balanced Manufacturing [18].

\section{Interfaces}

Cubes consolidate all information and resource flows (energy, materials, etc.) within clearly defined system boundaries, described as inputs and outputs. This approach not only promotes transparency during simultaneous analysis of energy and material flows, but the obtained modularity also increases flexibility for adaptation to specific environmental conditions. Cubes have uniformly and consistently defined interfaces, through which they interact with each other by exchanging energy, material and information flow, see Figure 4. The material flow incorporates the immediate value stream (e.g. workpiece, baking goods) and is described via discrete entities. All necessary energy flows (electrical, thermal, etc.) are represented as continuous variables together with their respective $\mathrm{CO}_{2}$ and cost rates and are quantified inside the cube boundaries using balance equations. Information flows provide operating states and monitoring values for the higher-level control as well as control actions for the cube module.

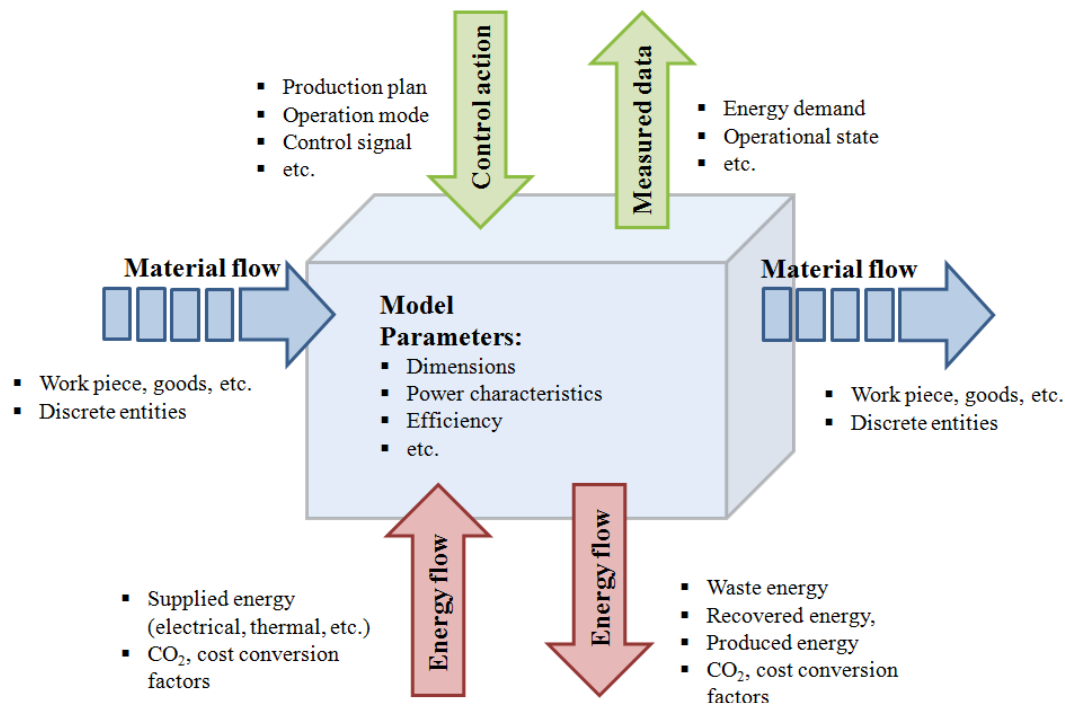

Figure 4. Generic cube interfaces with energy, material and information flows 


\section{Continuous and discrete flows}

The direct value stream is modelled via discrete entities. This is important for the traceability of individual products within the system boundaries and for assigning them product-related characteristics like the product footprint. Entities have a unique identity and store product-specific information in the form of attributes.

Continuous energy flows bearing energy, cost and $\mathrm{CO}_{2}$ emission footprints have to be aggregated in order to add their impact to a product's footprint. This is done via $\mathrm{CO}_{2}$ and cost density flows that go alongside with every energy flow. To get, for example, the total gram- $\mathrm{CO}_{2}$ of an energy flow during a given time period one must multiply the energy flow and the $\mathrm{CO}_{2}$ density flow and integrate the result over that time period. At the departure of the product the total $\mathrm{CO}_{2}$ value is added to the products $\mathrm{CO}_{2}$ footprint.

\section{Data collection and quality}

The quality of the product footprint quantification results relies heavily on the quality of the data used for the study. The bottom-up approach investigating value flows between cubes sets the requirement of detailed data aggregation. This is achieved through physical monitoring of the flows or via calculations that derive from detailed simulation models serving as virtual representations of the cubes and their interfaces. The optimum correlation of the two data acquisition paths will provide a solid database for the footprint calculations.

In accordance with the ISO 14067 standard, collected data, whether measured, calculated or estimated, are utilized to quantify the inputs and outputs of a unit process, hence a cube. Cube-specific data should be collected on site for individual processes that are included in the analyzed system. It is also possible to use literature data, estimates or other representative data according to the ISO standard. These non-site-specific data should be obtained and used carefully, always with documentation of the sources, assumptions and calculation methods.

Important aspects that should always be considered in order to preserve the quality of the whole process are the following:

- Age of data;

- Data precision and completeness;

- Representativeness and consistency;

- Uncertainty of the information.

\section{Footprint calculation}

There are three key phases during the manufacturing process and all products, by-products, raw materials and waste must always be located at one of the three during their stay within the system boundary. The three phases are production, transport and storage. Parameters, qualities and details about the key phases are provided in the following sections, as well as the method for calculating the footprint for energy systems and building functions.

\section{Allocation}

Allocation is the distribution of the footprint to different products and waste in a shared unit process. ISO 14067 determines that whenever possible allocation should be avoided by dividing the process in question or by expanding the system. If this is not possible the shares of burden should be distributed reflecting physical relationships between the products. Only if such physical relationships cannot be established, other factors, such as economic indicators may be used. However, there is an ongoing debate 
within the life-cycle assessment community about adequacy of subdivision, system expansion and allocation. It has been concluded that in most cases allocation cannot be entirely avoided by subdivision and system expansion [19], but can be problematic since it does not consider in how far the change in amount of co-products affects the output of the process [20]. Concerning allocation the pros and cons of physical vs. economic allocation have been the subject of an extensive discussion, which was conveniently summarized by [21]. [22] concludes that wherever physical causalities between functional units exist, allocation should be based on these causal relationships, however, that in some cases socio-economic demands are the driving force behind multiple-functions systems and therefore economic indicators best reflect the changes in the unit processes. [23] agrees that physical or functional causality should be the first principle applied in order to ensure fair allocation, followed by enviro-economic parameters.

Taking the discussed considerations into account and adjusting them for the proposed bottom-up approach, we suggest the following procedure for handling of co-products. As a general approach, the need for allocation should be avoided by splitting a cube into more detailed ones (subdivision). Furthermore, since the scope of the proposed method not only lies in reporting of a meaningful product footprint but also steering of the productions energy demand, we encourage not to limit the scope only to one product system but consider the entire available sphere of influence i.e. the whole company with all products. This system expansion ensures that all environmental burdens of co-products within the company are attributed to the correct products. Furthermore, it significantly limits the number of allocation problems, the remaining of which shall be discussed below.

Concerning the proposed allocation methods principles of causality and fairness were applied as well as considerations of feasibility. To differentiate the amount of footprint a product receives weight attributes are used. The suggested weighing factors try to reflect the causality of what causes the expenditures, which in some cases have been identified as physical or functional in other cases as economical. Feasibility considerations take into account, that the proposed method is designed for real-time evaluation of a batch or even single product i.e. analysis must be fairly swift to conduct. This limits the possibility to take factors outside the company into account to a certain extent. E.g. system expansion or elaborate allocation methods for waste leaving the system as analyzed by [24] or [25] are not feasible on a day-to-day basis. Therefore, more easily accessible factors must be found while still maintaining a certain amount of fairness of distribution. Based on these considerations three weights are defined.

Area weights. Area weights are used for assessing the cost of space in the production facility. For example, heating or lighting costs scale with the size of a production hall. Therefore the more room a product uses during transport or storage, respectively the bigger a production machine needs to be to work the product, the higher the footprint share of that product. Area weights reflect that the space requirements of production processes determine the size of the production facility and therefore the required resources to operate the space.

Market value weights. For the evaluation of the cost of work of different products the market value weight is defined. The market value of a product is the price that can be achieved by selling it on the free market. This weight is useful for differentiating main products from side products or waste. Other possible weights like product mass do not achieve a similar categorization and can lead to waste with high footprint shares in 
respect to the actual product. The assessment of the worth of semi-products can be difficult; the value gain in regards to the resource materials can be a valid indicator. Market values therefore represent a maybe not entirely truthful but feasible indicator.

Added value weights. The added value weight describes the value a product has gained during the entire stay inside the production plant. It is needed for assessing the contribution of a product to the general expenses of the plant. Examples are the administration of the company or research and development costs. This weight reflects that the economic benefit generated by a product is the cause of these processes.

\section{Production}

The first key phase addressed is the production phase. A product is considered in the production phase when it enters a production cube until it exits it again. During the production, necessary energy flows for the working process are aggregated and added upon exit to a product's footprint. The outputs of a production cube can carry a footprint only if they have a market value and are either sold or used later on. This applies likewise to material and energy flows. The share of footprint on output is assigned via market value weights. If no product is being worked in the production cube, the machine is either off, in standby or in setup or ramp-up state. If the machine is off no energy is being used and no footprint is aggregated. In standby the machine is using energy which has to be considered. Setup and ramp-up times are occurring when a machine is preparing to work the next batch of products.

Waste. Waste with no resale value cannot carry any footprint. For waste with disposal cost, the market value can be negative. Therefore additional footprint necessary for the transport, treatment and disposal has to be considered.

The production discard is incorporated at the location it is measured in the real system, creating a junction in the value flow. The allocation of the footprint is equivalent to that of waste.

Standby times. Footprint values for standby times during the production process are aggregated and handed to the next cube higher in the hierarchy; the thermal zone or building cube.

Setup and ramp-up times. The footprint resulting from setup and ramp-up times is caused by the products that are going to be produced next on that machine. Therefore the footprint is aggregated and stored until the batch of products is produced and then added. If allocation is necessary the market value weights is used. If at the end of the period under review a footprint is not assigned to a product, it is distributed between all products, again using the market value as weights.

\section{Transport}

The second key phase is the transport phase. Transport describes the transfer of one or more products from one place to another. The aggregation of the footprint due to the energy investment for the transport is equal to the procedure of the production. If different products are transported at the same time, the previously presented allocation method using market value weights applies.

However a special case occurs when considering the treatment of transport cubes. Since the aim of transport is to transfer products from one place to another, it is possible that borders of thermal zones are being crossed. This poses a problem because the cube 
concept is strictly hierarchical and one cube cannot be contained in two thermal zones. To be consistent with the approach, it is therefore necessary, to describe and model the transport to the border of the thermal zone in one cube and the transport in the next thermal zone in another cube. If the transport system in question is rather flexible (e.g. a forklift) the number of transport cubes necessary for the description can be unreasonably large. It is advisable to use constraints to reduce the number of possible paths and reduce the system complexity.

\section{Storage}

The third key phase is storage. Here the treatment of the footprint is similar to the transport cubes but simplified due to the absence of the ability of the products to move. The storage can have an energy demand (e.g. an automated storage system) that has to be aggregated to the stored products. Otherwise the storage cube only represents the time delay between a product entering and exiting the cube due to it being stored.

\section{Energy systems}

Energy systems are handled with an approach similar to the method for treating the production cubes. The difference is that the products never get in direct contact with the energy system. Therefore the footprint entering the energy system through the drawn energy is not added to any product directly, but rather forwarded with the usable energy output. Ultimately, this output energy flow will either lead into a production or thermal zone cube, where the footprint is added to the product. For example in case of a compressor, the footprint of the input electricity is forwarded to the output compressed air. This air is connected to a machine used for a work process where the footprint of the compressed air is added to the product.

\section{Thermal zones and building}

The effect of the building HVAC and lighting systems on products is addressed throughout the three key phases of the manufacturing process. The evaluation of HVAC and lighting effect on a product's footprint value occurs through the actual required area, based on area weights, for a product in each of the three key phases.

Area weights are used for assessing the cost of space in the production facility, needed for heating, cooling, air conditioning and lighting. During the stay inside the system boundary a product is either in production, storage or transport. The production area is defined as the area a machine occupies. The storage area is the area that a product uses when in storage. Different types of storage methods need to be assessed in regards to the space they are using. For example rack storage, silos and tanks need to be compared. Finally, the transport area is the space needed for transporting a product. The assessment of transport technologies like cranes, forklifts, trucks or conveyor belts is grounded on the area reserved for products to be transported.

The product footprint is evenly distributed to the products using these area weights. The value of an area is a characteristic of a product and it may change through different production steps. For mixed storage (e.g. shelves, silos and pallets) unit conversion factors must be considered between the different types. In case footprint from HVAC and lighting in a thermal zone occurs, but no product is present, the footprint value is forwarded to the next highest cube in the hierarchy (e.g. building) and distributed using area weights.

The building cube does not have energy demand by itself and only functions as collector of footprint otherwise not assignable. This footprint can stem from thermal zones without products in them (see above), the administration or auxiliary system cubes 
(see below). The footprint of the building is distributed to all the products inside the building using the added value weight. In case the building itself is empty at some point during the examined time period the footprint is aggregated and stored until the end of the time period. It is then retroactively distributed to all products that have been in the system during the analysis period.

\section{Administration and auxiliary systems}

Further attention must be given to the footprint created by services and systems not directly linked to the manufacturing process. In this category energy and resources consumed by auxiliary systems, administrative services or similar processes are taken into account. Examples can be desktop computers, servers, printer, kitchens, research labs and many more. In respect to ISO 14067 it is required to justify decisions of disregarding energy consumers hence $\mathrm{CO}_{2}$ and costs impacts. The footprint generated from administration and auxiliary systems is forwarded to the highest hierarchy level and there it is distributed using added value weights.

\section{RESULTS AND DISCUSSION}

To demonstrate the presented methods, the procedure of analyzing the production plant and conducting the footprint calculation is applied to a case study. The production plant under consideration consists of a building with an oven for annealing metal parts. Two different products, called A and B, can be treated using the machine. However, for switching products a setup has to be performed. After the annealing process the products are examined and defective parts are discarded as waste. The remaining products are moved by a conveyor belt to a storage room. In a third room equipment for heating and cooling is located. A gas heater is used for heating the oven and the storage room. For reaching high temperatures the oven is additionally heated electrically. A compression chiller is cooling the production hall.

The facility operates seven days a week, therefore the choice of time boundaries is more flexible. For simplicity sake the time boundary is chosen to be one day. The system boundary is the building. The task is to calculate the product carbon footprint for the products produced in the facility during the time period for a given scenario.

To analyze the system, a cube model is derived from the system description, as seen in Figure 5. The plant consists of a building cube, which contains three thermal zone cubes. The annealing oven and conveyor belt are shown in the first thermal zone, the storage room in the third. The energy system cubes are situated in the second thermal zone. Additionally to the already mentioned system, an electric network cube is needed for distributing electric power. Three providers are sources for feeding inputs to the system, from top to bottom: natural gas (energy flow), electric energy (energy flow) and raw materials (material flow). The sinks called Customer and Waste receive entities after leaving the system. The belt cube does not require energy. This is a simplification justifiably by its low energy demand.

The initial footprint values of raw materials coming from the provider are zero since we are only interested in the partial footprint produced by the production facility. The footprint densities of the energy flows at the system boundary (providers) are supplied by the local energy provider.

In the next step the weights are determined. The area weights, denoted by $w_{\mathrm{ar}, X, C}$, have to be defined for both products $\mathrm{A}$ and $\mathrm{B}$ (index $X$ ) and for all the cubes they can enter (index $C$ ), six in total. The waste does not require a weight to be defined since it exists only at the departure of the machine and then leaves the system immediately. 


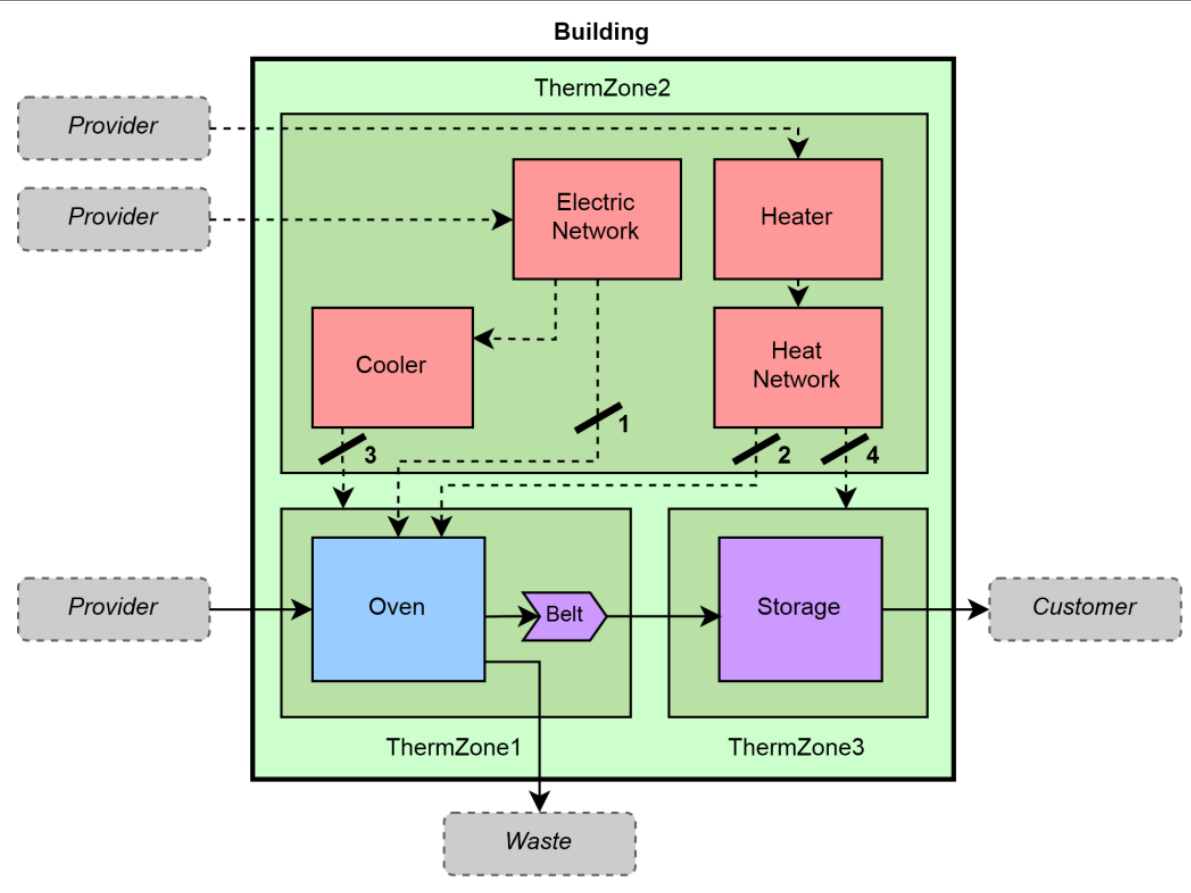

Figure 5. Example plant for product manufacturing carbon footprint analysis. The interfaces " 1 ", "2", "3" and "4" correspond to the energy flow footprints shown in Table 1

Table 1. Product footprint calculation; all values in $\left[\mathrm{g} \mathrm{CO}_{2}\right]$

\begin{tabular}{|c|c|c|c|c|c|c|c|c|c|c|c|c|}
\hline Event & Time & & Energy & low footp & rint & All & Bat & ch type A & & Batch & ype B & \\
\hline & & 1 & 2 & 3 & 4 & & P1 & P2 & & P3 & & Waste \\
\hline to & 00:00 & 0 & 0 & 0 & 0 & & & & & & & \\
\hline $\mathrm{t} 1$ & $00: 35$ & 0 & 0 & 4,160 & 5,859 & 10,019 & & & & & & \\
\hline $\mathrm{t} 2$ & 01:02 & 52 & 542 & 3,428 & 4,520 & 7,949 & & & 594 & & & \\
\hline $\mathrm{t} 3$ & $04: 32$ & 305 & 843 & 25,508 & 35,261 & & 61,918 & & & & & 0 \\
\hline $\mathrm{t} 4$ & $04: 35$ & 4 & 12 & 346 & 505 & & 332 & 535 & & & & \\
\hline $\mathrm{t} 5$ & 08:05 & 300 & 843 & 25,221 & 35,418 & & 35,418 & 26,364 & & & & 0 \\
\hline t6 & 08:08 & 5 & 60 & 324 & 504 & & 504 & 324 & & & 65 & \\
\hline $\mathrm{t} 7$ & 09:15 & 106 & 1,345 & 7,014 & 11,246 & & 9,130 & 9,130 & & & 1,451 & \\
\hline t8 & $13: 25$ & 486 & 4,014 & 17,895 & 41,414 & & 20,707 & 20,707 & & 22,395 & & 0 \\
\hline t9 & $13: 28$ & 0 & 0 & 162 & 492 & & 246 & 246 & & 162 & & \\
\hline $\mathrm{t} 10$ & 14:03 & 4 & 0 & 1,882 & 5,744 & & 2,065 & 2,065 & & 3,500 & & \\
\hline $\mathrm{t} 11$ & $23: 59$ & 0 & 0 & 55,707 & 98,400 & 154,108 & & & & & & \\
\hline Tend & & & & & & & 57,655 & 57,655 & & 58,874 & & \\
\hline Sum & & 1,263 & 7,658 & 141,647 & 239,364 & & 187,975 & 117,026 & & 84,932 & & \\
\hline Total & & & 389,9 & & & & & & 9,932 & & & \\
\hline
\end{tabular}

The area weight for the products while being worked at (i.e. in the oven) is the base area of the machine, $10 \mathrm{~m}^{2}$ in this example, and identical for both products. The area weight while on the conveyor belt depends on how much space the products occupy while being transported. In this example product A and B occupy $3 \mathrm{~m}^{2}$ each. In the storage room the area weight depends on the space required for storing a product. It is possible to stack product $\mathrm{A}$ up to four times, while this is not possible for the smaller product B. Therefore the area weight while in storage of product $\mathrm{A}$ is only $0.5 \mathrm{~m}^{2}$.

The market value weights $w_{\mathrm{mv}, X}$ have to be established in a similar way. The market value weight is used for the footprint allocation at a machine or logistics cube, thus the 
market value has to be determined for the product after being worked on. The market value for the smaller product B turns out to be considerably higher due to a more intricate design of the piece. In this scenario the market value weight of product B is EUR 3,000, three times that of product A. Since neither of the products gains value from transport or storage, the market value weights are identical for all three cubes the products visit. The market value weight of the waste is zero since it cannot be sold and does not produce significant cost for disposal.

The added value weight $w_{\mathrm{av}, X}$ assesses the added value of a product through its production in the system. In the example both product A and B receive the same treatment in the oven, thus the added value weight is the same. Again, because the waste does not stay in the system, no added value weight needs to be defined for it.

For the calculation of the product footprint the necessary data has to be compiled, both for the energy flows and the material flow. Regarding the material flow, it has to be known when a product enters or exits a cube. For the continuous flows the power trajectories over time for energy flows for cubes in direct contact with the products have to be available, as well as the associated $\mathrm{CO}_{2}$ densities. The data can be obtained from a monitoring system or calculated (e.g. via a simulation model as shown in [17]).

\section{Product footprint calculation}

The calculation of the product manufacturing carbon footprint is shown by progressing through the considered time period of one day, from start to finish. The production plan of the day consists of one batch of two products of type A and another batch containing one product of type B. When an event occurs, i.e. something that affects the footprint calculation changes (e.g. entity entering or leaving, weight changing) the time period between the last event and the current one has to be evaluated.

Table 1 shows the results of the calculation. At the far left the name of the event and the respective timestamp is shown. The next columns represent the aggregated footprint of energy flows $f_{\mathrm{EF}}$ between the previous event and the current one with the number of the energy flow correlating to the interfaces marked in Figure 5. The blue colour indicates the input flows of the oven, respectively green for the energy demand of the thermal zones. Eq. (1) shows the calculation of the energy flow footprints, where $P$ is the energy flow in $\mathrm{W}$ and $\rho_{\mathrm{CO} 2}$ is the corresponding carbon footprint density in $\mathrm{g} \mathrm{CO}_{2} / \mathrm{J}$ :

$$
f_{\mathrm{EF}, i}=\int_{t_{i-1}}^{t_{i}} P(t) \rho_{\mathrm{CO} 2}(t) d t
$$

For the evaluation of the equation the trajectories of energy flows and densities of the cubes in direct contact with the product are necessary. In the example these cubes are the oven (with two inputs), thermal zone 1 and thermal zone 3 (each one input). Although belt, storage and building are in contact with products as well they do not have energy flow inputs or outputs and are therefore disregarded. This leaves four energy flow trajectories and their footprint densities, as shown in Figure 6 and Figure 7 respectively. The densities for the heat of the oven and thermal zone 3 are identical and only listed once.

The right side of Table 1 shows where the footprint is aggregated onto. The column All contains footprint that cannot be attributed to entities immediately and is therefore stored to be attributed at the end of the calculation, indicated by the red text colour. The next columns contain the footprint aggregated to specific products and a column for 
footprint to be stored and distributed to a certain batch. Finally a column for the waste footprint is reserved.

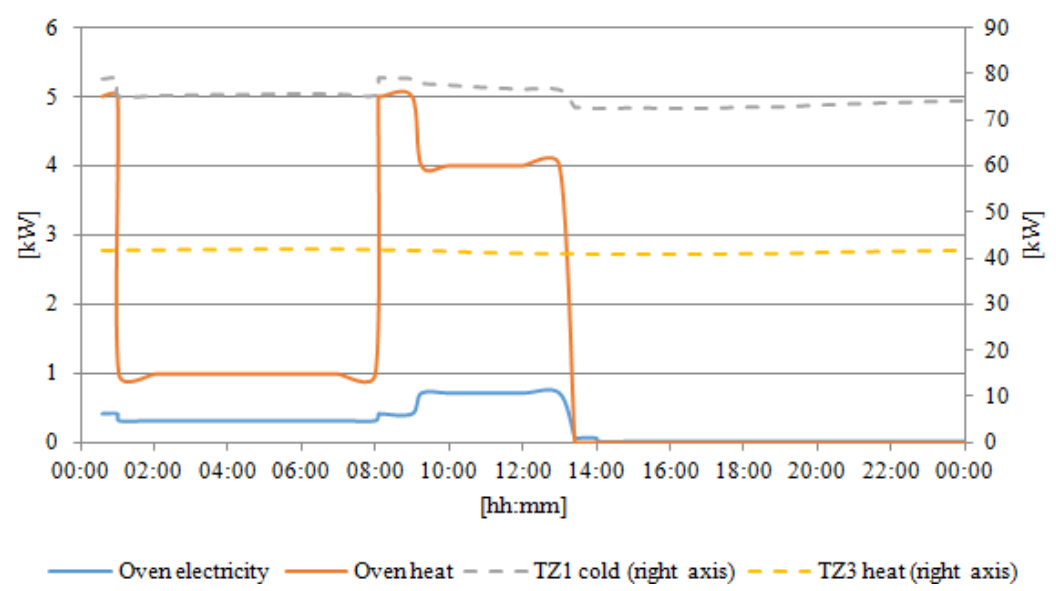

Figure 6. Energy flows relevant for footprint calculation of the given scenario

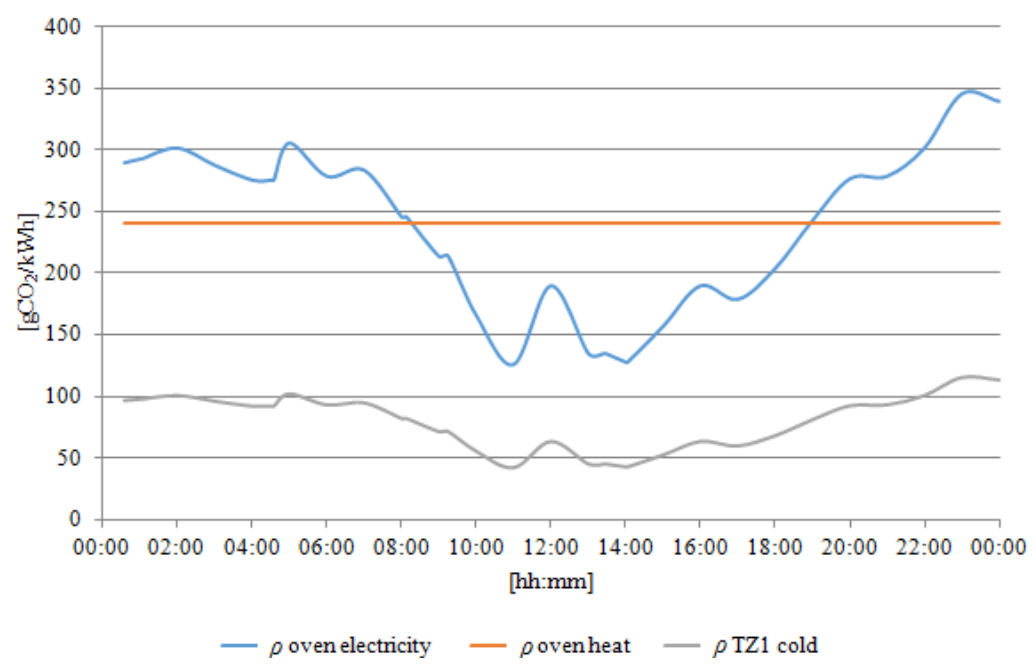

Figure 7. Carbon footprint densities of the energy flows of Figure 6

The rows of Table 1 show the events. To clarify further, the events are discussed in detail. For brevity, repeated calculations are not shown.

t0. At the start of the day no products are inside the plant and the oven is off. The only energy demand stems from heating of thermal zone 3 and cooling of thermal zone 1 .

t1. The first event is the oven being turned on and entering setup to produce a batch of two products of type A. Since no product was in either of the thermal zones, the footprint aggregated up until now (from thermal zones, i.e. interfaces 3 and 4) is forwarded to the higher hierarchy level, i.e. the building. Since no products were in the building either and it is the highest level in the hierarchy, the footprint is aggregated and stored. Therefore the footprint contribution of the building at event $\mathrm{t} 1, F P_{\mathrm{All}, \mathrm{t} 1}$, is shown in eq. (2):

$$
F P_{\mathrm{All}, \mathrm{t} 1}=f_{3, \mathrm{t} 1}+f_{4, \mathrm{t} 1}
$$

t2. After the setup is completed the first product of type A, called P1, enters the oven. The treatment of heating and cooling footprint aggregated up until now stays the same. The 
footprint of the machine for setup (from electrical and thermal energy, i.e. interfaces 1 and 2) is aggregated and stored for adding it at the end of the day to the two products of the batch:

$$
F P_{\mathrm{BA}, \mathrm{t} 2}=f_{1, \mathrm{t} 2}+f_{2, \mathrm{t} 2}
$$

t3. The next event is that product P1 and waste leave the oven, product P1 enters the conveyor belt, and the waste leaves the system. At this point the footprint of the oven for producing the first product is aggregated and added to product P1 and the waste using the market value weights. Since the market value weight of the waste is zero in this scenario, the entire footprint is assigned to P1. At the same time another product of type A, called P2, enters the oven. The cooling demand of thermal zone 1 is added to the footprint of product $\mathrm{P} 1$ since it was the only product in that zone. The heating demand of thermal zone 3 is forwarded to the building and there assigned to product P1. Eq. (4) shows the calculation of the footprint of product $\mathrm{P} 1$ :

$$
F P_{\mathrm{P} 1, \mathrm{t} 3}=\left(f_{1, \mathrm{t} 3}+f_{2, \mathrm{t} 3}\right) \frac{w_{\mathrm{mv}, \mathrm{A}}}{w_{\mathrm{mv}, \mathrm{A}}+w_{\mathrm{mv}, \mathrm{w}}}+f_{3, \mathrm{t} 3}+f_{4, \mathrm{t} 3}
$$

t4. The next time interval ends when P1 exits the conveyor, exits thermal zone 1 and enters thermal zone 3 and the storage. During that time period two products were in thermal zone 1, thus the footprint for cooling needs to be distributed using the area weights of oven (containing P1) and belt (holding P2). The footprint distributed by the building is added to both products using the added value weights of product P1 and P2. With them being identical, they both get equal shares:

$$
\begin{gathered}
F P_{\mathrm{P} 1, \mathrm{t} 4}=f_{3, \mathrm{t} 4} \frac{w_{\mathrm{ar}, \mathrm{A}, \mathrm{Belt}}}{w_{\mathrm{ar}, \mathrm{A}, \mathrm{Belt}}+w_{\mathrm{ar}, \mathrm{A}, \mathrm{Oven}}}+f_{4, \mathrm{t} 4} \frac{w_{\mathrm{av}, \mathrm{A}}}{2 w_{\mathrm{av}, \mathrm{A}}} \\
F P_{\mathrm{P} 2, \mathrm{t} 4}=f_{1, \mathrm{t} 4}+f_{2, \mathrm{t} 4}+f_{3, \mathrm{t} 4} \frac{w_{\mathrm{ar}, \mathrm{A}, \mathrm{Oven}}}{w_{\mathrm{ar}, \mathrm{A}, \mathrm{Belt}}+w_{\mathrm{ar}, \mathrm{A}, \mathrm{Oven}}}+f_{4, \mathrm{t} 4} \frac{w_{\mathrm{av}, \mathrm{A}}}{2 w_{\mathrm{av}, \mathrm{A}}}
\end{gathered}
$$

t5. When product P2 and its waste exit the oven, the next time period ends. Product P2 enters the conveyor, the waste leaves the building. The oven enters setup for producing a batch of one product type B. Since product P1 was in storage the footprint for heating thermal zone 3 is aggregated to that product and not forwarded to the building, resulting in the building not distributing any footprint. The footprint for cooling thermal zone 1 is added to product $\mathrm{P} 2$.

t6. Product P2 exits the conveyor and enters the storage room. Product P2 was in thermal zone 1, the footprint for cooling is again added to it. The footprint for heating thermal zone 3 is added to product $\mathrm{P} 1$.

t7. When setup is finished, a product of type B, called P3, enters the oven. The footprint accumulated during setup is stored for later adding it to the product of the second batch. In the previous time period no product was located in thermal zone 1 , therefore the footprint is forwarded to the building where it is distributed to products P1 and P2 using added value weights. Thermal zone 3 now has two products and the footprint is added to them using area weights.

t8. When P3 is finished, it exits the oven and enters the conveyor. No waste is produced. The oven enters standby. During the time period the footprint of thermal zone 1 was not forwarded but directly added to P3. 
t9. Product P3 exits the conveyor and enters storage in thermal zone 3. The standby power consumption of the oven during this time period is forwarded to the next hierarchy level (thermal zone 1) and there added to P3.

t10.The last event of the day is the oven being turned off and the three products being removed from storage and leaving the system. Since no product was in thermal zone 1, the footprint is forwarded and assigned by the building using added value weights. Thermal zone 3 distributes the footprint for heating to all products inside using area weights for storage.

t11.The examination time period ends. In the last segment no products were located inside the system. Like in the beginning, all footprint is forwarded to the building and stored.

At the very end, the stored footprint is distributed. The footprint stored by the building is distributed using added value weights between products P1, P2 and P3. The footprint stored from the first setup is added to products P1 and P2. The footprint stored because of setup for batch two is added to product P3. Eq. (7) illustrates the final calculation of the footprint for P1:

$$
\begin{gathered}
F P_{\mathrm{P} 1}=\sum_{i=\mathrm{t}_{1}}^{\mathrm{t}_{11}} F P_{\mathrm{P} 1, \mathrm{i}}+\left(F P_{\mathrm{All}, \mathrm{t} 1}+F P_{\mathrm{All}, \mathrm{t} 2}+F P_{\mathrm{All}, \mathrm{t} 11}\right) \frac{w_{\mathrm{av}, \mathrm{A}}}{2 w_{\mathrm{av}, \mathrm{A}}+w_{\mathrm{av}, \mathrm{B}}}+ \\
+F P_{\mathrm{BA}, \mathrm{t} 2} \frac{w_{\mathrm{av}, \mathrm{A}}}{2 w_{\mathrm{av}, \mathrm{A}}}
\end{gathered}
$$

In the end, all footprints passing through the system boundary with the energy flows are assigned to a product, no footprint is lost, as seen in the last row of Table 1. It also shows the importance of a bottom up approach: The footprint received by the three products is significantly different. Compared to a traditional top-down approach P1 receives $45 \%$ more footprint, mainly due to the high cost of cooling while being alone in the facility. This shows that the presented method achieves a high spacial and temporal resolution of the footprint per product.

In this example only the footprint generated inside the system was of interest. To gain a lifecycle footprint, other facilities necessary for production need to be investigated as well as estimates for the footprint accumulation during the other lifecycle phases need to be done, thereby expanding the results of the partial footprint.

\section{CONCLUSIONS}

The presented method for aggregating a product footprint is designed to fairly distribute the expenditures of a production plant to the manufactured products. Although care has been taken at intercepting special problems, not all possible constellations appearing at a plant can be easily addressed. The first obvious risk is the choice and assertion of weights for allocation. Even though the total amount of footprint is conserved, the distribution can vary heavily. Therefore the attribution of weights to different products has to be done carefully to avoid errors. Secondly, thermal energy stored in workpieces can have an influence on the surrounding thermal zone, resulting in additional thermal loads. It can be argued that these loads do carry a footprint since the product is responsible for higher or lower heating or cooling in that zone. However, due to the high complexity of the calculation and in most cases insignificant influence, the effect is being neglected.

In conclusion, a generic method for aggregating a time, cost, energy and carbon emission product footprint (more specifically the dynamic product manufacturing footprint) has been presented. Special attention has been provided for typical scenarios 
that occur in production plants, independent of sector of industry. The presented method can help in providing detailed feedback about the ecologic and economic impact of a manufacturing plant. This can be used for comparing different production strategies and further to optimize the plant operation.

\section{ACKNOWLEDGEMENT}

We gratefully acknowledge the work of all project-partners of Balanced Manufacturing. The research presented is funded by the Austrian Climate and Energy Funds within the program e!MISSION.at - Energy Mission Austria.

\section{REFERENCES}

1. Eurostat, Energy Balances, http://ec.europa.eu/eurostat/web/energy/data/ energy-balances, [Accessed: 22-July-2015]

2. Bonneville, E. and Rialhe, A., Good Practice for Energy Efficiency in Industry, 2006, https://leonardo-energy.org/sites/leonardo-energy/files/root/Documents/2009/DSMindustry.pdf, [Accessed: 29-April-2016]

3. Kim, H. C., Keoleian, G. A. and Horie, Y. A., Optimal Household Refrigerator Replacement Policy for Life Cycle Energy, Greenhouse Gas Emissions and Cost, Energy Policy, Vol. 34, No. 15, pp 2310-2323, 2006, http://dx.doi.org/10.1016/j.enpol.2005.04.004

4. Kozioł, J. and Mendecka, B., Evaluation of Economic, Energy-environmental and Sociological Effects of Substituting Non-renewable Energy with Renewable Energy Sources, J. of Sustain. Dev. Energy, Water Environ. Syst., Vol. 3, No. 4, pp 333-343, http://dx.doi.org/10.13044/j.sdewes.2015.03.0025

5. Moriarty, P. and Wang, S., Eco-Efficiency Indicators for Urban Transport, J. of Sustain. Dev. Energy, Water Environ. Syst., Vol. 3, No. 2, pp 183-195, http://dx.doi.org/10.13044/j.sdewes.2015.03.0015

6. Song, G., Che, L. and Zhang, S., Carbon Footprint of a Scientific Publication: A Case Study at Dalian University of Technology, China, Ecol. Indic., Vol. 60, pp 275-282, 2016, http://dx.doi.org/10.1016/j.ecolind.2015.06.044

7. Liu, Y., Dynamic Study on the Influencing Factors of Industrial Firm's Carbon Footprint, J. of Clean. Prod., Vol. 103, pp 411-422, 2015, http://dx.doi.org/10.1016/j.jclepro.2014.06.029

8. Padgett, J. P., Steinemann, A. C., Clarke, J. H. and Vandenbergh, M. P., A Comparison of Carbon Calculators, Environ. Impact Assess. Rev., Vol. 28, No. 2-3, pp 106-115, 2008, http://dx.doi.org/10.1016/j.eiar.2007.08.001

9. Gaussin, M., Hu, G., Abolghasem, S., Basu, S., Shankar, M. R. and Bidanda, B., Assessing the Environmental Footprint of Manufactured Products: A Survey of Current Literature, Int. J. Prod. Econ., Vol. 146, No. 2, pp 515-523, 2013, http://dx.doi.org/10.1016/j.ijpe.2011.12.002

10. Neugebauer, R., Westkämper, E., Klocke, F., Kuhn, A., Schenk, M., Michaelis, A., Spath, D. and Weidner, E., Untersuchung zur Energieeffizienz in der Produktion Abschlussbericht, Chemnitz, 2008.

11. Wiedmann, T. and Barrett, J., A Review of the Ecological Footprint Indicator Perceptions and Methods, Sustainability, Vol. 2, No. 6, pp 1645-1693, 2010, http://dx.doi.org/10.3390/su2061645

12. Herrmann, C., Thiede, S., Kara, S. and Hesselbach, J., Energy Oriented Simulation of Manufacturing Systems - Concept and Application, CIRP Ann. - Manuf. Technol., Vol. 60, No. 1, pp 45-48, 2011, http://dx.doi.org/10.1016/j.cirp.2011.03.127

13. Fang, K., Uhan, N., Zhao, F. and Sutherland, J. W., A New Approach to Scheduling in Manufacturing for Power Consumption and Carbon Footprint Reduction, J. of 


Manuf. Syst., Vol. 30, No. 4, pp 234-240, 2011,
http://dx.doi.org/10.1016/j.jmsy.2011.08.004

14. Messagie, M., Mertens, J., Oliveira, L., Rangaraju, S., Sanfelix, J., Coosemans, T., Van Mierlo, J. and Macharis, C., The Hourly Life Cycle Carbon Footprint of Electricity Generation in Belgium, bringing a Temporal Resolution in Life Cycle Assessment, Appl. Energy, Vol. 134, pp 469-476, 2014, http://dx.doi.org/10.1016/j.apenergy.2014.08.071

15. Lindskog, E., Lundh, L., Berglund, J., Tina Lee, Y., Skoogh, A., Johansson, B. and Johansson, B., A Method for Determining the Environmental Footprint of Industrial Products using Simulation, Proceedings of the 2011 Winter Simulation Conference (WSC), pp 2131-2142, 2011, http://dx.doi.org/10.1109/WSC.2011.6147926

16. Bunse, K., Vodicka, M., Schönsleben, P., Brülhart, M. and Ernst, F. O., Integrating Energy Efficiency Performance in Production Management - Gap Analysis between Industrial needs and Scientific Literature, J. of Clean. Prod., Vol. 19, No. 6-7, pp 667-679, 2011, http://dx.doi.org/10.1016/j.jclepro.2010.11.011

17. Leobner, I., Smolek, P. Heinzl, B., Kovacic, I., Ponweiser, K., Mayrhofer, W., Kastner, W. and Dür, F., Balanced Manufacturing - A Methodology for Energy Efficient Production Plant Operation, Proceedings of the $10^{\text {th }}$ Conference on Sustainable Development of Energy, SDEWES2015.0268, pp 1-11, 2015.

18. Projekt Balanced Manufacturing, http://bama.ift.tuwien.ac.at/, [Accessed:
[ 29-April-2016]

19. Ekvall, T. and Finnveden, G., Allocation in ISO 14041 - A Critical Review, J. of Clean. Prod., Vol. 9, No. 3, pp 197-208, 2001, http://dx.doi.org/10.1016/S0959-6526(00)00052-4

20. Weidema, B. P. and Schmidt, J. H., Avoiding Allocation in Life Cycle Assessment Revisited, J. Ind. Ecol., Vol. 14, No. 2, pp 192-195, 2010, http://dx.doi.org/10.1111/j.1530-9290.2010.00236.x

21. Ardente, F. and Cellura, M., Economic Allocation in Life Cycle Assessment, J. of Ind. Ecol., Vol. 16, No. 3, pp 387-398, 2012, http://dx.doi.org/10.1111/j.1530-9290.2011.00434.x

22. Azapagica, A. and Cliftb, R. Allocation of Environmental Burdens in Multiple-function Systems, J. of Clean. Prod., Vol. 7, No. 2, pp 101-119, 1999, http://dx.doi.org/10.1016/S0959-6526(98)00046-8

23. Frischknecht, R., Allocation in Life Cycle Inventory Analysis for Joint Production, Int. J. of Life Cycle Assess., Vol. 5, No. 2, pp 85-95, 2000, http://dx.doi.org/10.1007/BF02979729

24. Nicholson, A. L., Olivetti, E. A., Gregory, J. R., Field, F. R. and Kirchain, R. E., End-of-life LCA Allocation Methods: Open Loop Recycling Impacts on Robustness of Material Selection Decisions, 2009 IEEE International Symposium on Sustainable $\begin{array}{llll}\text { Systems } \quad \text { Technology, 2009, } & \text { 1-6, }\end{array}$ http://dx.doi.org/10.1109/ISSST.2009.5156769

25. Johnson, J. X., McMillan, C. A. and Keoleian, G. A., Evaluation of Life Cycle Assessment Recycling Allocation Methods, J. of Ind. Ecol., Vol. 17, No. 5, 2013, http://dx.doi.org/10.1111/jiec.12050 